\title{
Study on Evaluation Indexes of Surgical Manipulations with a Stereoscopic Endoscope
}

\author{
Yasushi Yamauchi ${ }^{1}$ and Kazuhiko Shinohara ${ }^{2}$ \\ ${ }^{1}$ Surgical Assist Technology Group, National Institute of Advanced Industrial Science and \\ Technology (AIST), AIST Tsukuba Central 6, 1-1-1 Higashi, Tsukuba 305-8566, Japan \\ y.yamauchi@aist.go.jp, http://staff.aist.go.jp/y.yamauchi/ \\ ${ }^{2}$ School of Bionics, Tokyo University of Technology, \\ 1404 Katakura, Hachioji, Tokyo 192-0982, Japan \\ fwpa6707@mb. infoweb.ne.jp
}

\begin{abstract}
We compared stereoscopic displays (3D) with monocular displays (2D) by experimenting on the manipulation of forceps with a laparoscope. The task consisted of repetitive movements of the tip of the forceps under both conditions to targets. Time for manipulation with a 3D display was significantly shorter than for a 2D display. Little significant difference was observed for the psychological indexes.
\end{abstract}

\section{Introduction}

For medical stereoscopic imaging, the usefulness of the presentation of depth information and the issues of fatigue have been discussed. The report on an endoscopic trainer [1] revealed a remarkable improvement in the accuracy of forceps manipulation by a stereoscopic endoscope than a monocular endoscope. Clinical findings on cholecystectomy [2], however, revealed no difference in performance between them.

This study attempted to measure and to evaluate surgical manipulations performed by using a stereoscopic endoscope. Evaluation indexes consisted of a physical index (task execution time) and psychological indexes on depth perception.

\section{Methods}

We used a stereoscopic laparoscope (SK-1057-3D-A, Shinko Optical, Japan) and a forceps (5-mm EndoGrasp, AutoSuture). Stereoscopic (3D) images were presented in a $120-\mathrm{Hz}$ time-sharing display system and a polarized glasses. The system was also capable of providing $2 \mathrm{D}$ images by presenting the left (or right) images to both eyes.

24 subjects were asked to move a conductive rubber chip fixed on the end of the forceps. Ten numbered holes were randomly drilled on the surface of a curved pegboard (Fig. 1) placed inside a laparoscope trainer (Limbs \& Things). A target number was randomly displayed on a PC screen and the subject was required to insert the chip 
into the hole of the corresponding number. This procedure was repeated for five minutes in both $2 \mathrm{D} / 3 \mathrm{D}$ displays. The subject was not told which was being presented ('blind test').

For measurement of the psychological indexes, a questionnaire was conducted after the experiment, including a question of fatigue and the order of $2 \mathrm{D} / 3 \mathrm{D}$ displays.

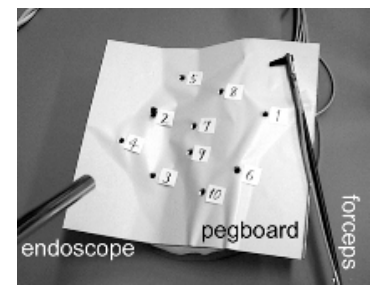

Fig. 1. Pegboard

\section{Results and Discussion}

As a performance index (Table 1), the ratio of the average time required in the first trial to the time in the second trial to manipulate the forceps was used. In Group B, the time for manipulation with a 3D display was shorter than for a 2D display. In contrast, Group A showed no statistically significant difference between two trials. This is probably because the subjects in Group A improved their manipulation performance by observing a 3D display to such an extent as to compensate for the learning effect.

Table 2 shows the results of the questionnaire investigation. Only in "Fatigue," significance was found, but the difference was small. 9 of the 24 subjects were not able to correctly discern a 3D image from a 2D image.

Table 1. Results on performance index

\begin{tabular}{cccc}
\hline Group (trial order) & \# of subjects & Avg. time per procedure (ratio) & \\
\hline A (3D before 2D) & 11 & 2D $/ 3 \mathrm{D}=0.88 \pm 0.19$ & $\mathrm{p}>0.10$ \\
B (2D before 3D) & 13 & 3D/2D $=0.69 \pm 0.17$ & $\mathrm{p}<0.01$ \\
\hline
\end{tabular}

Table 2. Questions and results on psychological indexes

\begin{tabular}{lccl}
\hline Questions (4:very strong 1:none) & 3D & 2D & \\
\hline Did you have a sense of depth? & 3.0 & 2.5 & $\mathrm{p}>0.05$ \\
Did you have a feeling similar to motion sickness? & 1.2 & 1.1 & $\mathrm{p}>0.05$ \\
Did you have eye fatigue? & 1.7 & 1.5 & $\mathrm{p}<0.05$ \\
\hline Which of the two trials involved 3D image presentation? & 15 & 5 & (\# of 24) \\
\hline
\end{tabular}

\section{References}

1. Taffinder, N., et al.: The effect of a second-generation 3D endoscope on the laparoscopic precision of novices and experienced surgeons. Surg Endosc 13 (1999) 1087-1092

2. Hanna, G.B., et al.: Randomised study of influence of two-dimensional versus threedimensional imaging on performance of laparoscopic cholecystectomy. Lancet 351 (1998) 248-251 